\title{
Anatomical criteria for the diagnosis of sinus venosus defects
}

Ahmed M Al Zaghal, Jia Li, Robert H Anderson, Christopher Lincoln, Darryl Shore, Michael L Rigby

\begin{abstract}
Background-The diagnosis of sinus venosus defects remains a matter of debate. It is crucial to provide solid anatomical criteria, by identifying the very nature of the atrial septum relative to sinus venosus defects, to diagnose and differentiate them from other interatrial communications.

Objective-This study was designed to reestablish the anatomical criteria for the diagnosis of sinus venosus defects.

Methods-Five specimens with sinus venosus defects from the cardiopathological museum were examined. Study of the abnormal hearts was supplemented by examining the extent and structure of the atrial septum in 10 normal hearts. The echocardiograms and surgical notes were reviewed from 18 patients seen between July 1991 and August 1996 at the Royal Brompton Hospital in London diagnosed preoperatively to have a sinus venosus defect.
\end{abstract}

Results-The nature of the oval fossa and its muscular borders were identified in the normal hearts. In all three autopsied specimens of the superior variety of sinus venosus defect, the mouth of the superior caval vein was overriding the intact muscular anterosuperior border of the oval fossa. Two specimens thought initially to have the inferior variety of sinus venosus defect were re-classified as having defects within the oval fossa as it was the deficient oval fossa itself, rather than its intact muscular border, that was overridden by the mouth of the inferior caval vein. Sixteen patients had been diagnosed echocardiographically as exhibiting the superior variant of the defect. Retrospective review showed overriding of the superior caval vein across the upper rim of the oval fossa in 12 patients. These findings were confirmed by surgery in 11 patients with the 12 th awaiting operation. Overriding of the fossa by the caval vein was not found in the other four patients. Surgery in all of these showed the defect to be within the oval fossa. In two patients diagnosed echocardiographically as having inferior defects, the surgical findings confirmed a biatrial connection of the inferior caval vein in one patient, the findings in the second were equivocal.

Conclusions-The key anatomical criterion for the diagnosis of sinus venosus defects is overriding of the mouth of the superior or inferior caval vein across the intact muscular border of the oval fossa. The interatrial communication is then formed within the mouth of the overriding vein, and is outside the confines of the oval fossa.

(Heart 1997;78:298-304)

Keywords: anatomical criteria; sinus venosus defect; nature of the atrial septum; interatrial communication

So-called sinus venosus defects are relatively uncommon forms of interatrial communication. Superior and inferior varieties are recognised related to the superior and inferior caval veins, respectively, with the inferior variant being particularly rare. Although previous studies have suggested biatrial connection of one of the caval veins as a diagnostic criterion, extraseptal location of the interatrial communication, along with anomalous pulmonary venous drainage, are held to be the basic anatomical features. ${ }^{1-4}$ More recently, however, others have questioned these findings. ${ }^{5} 6$

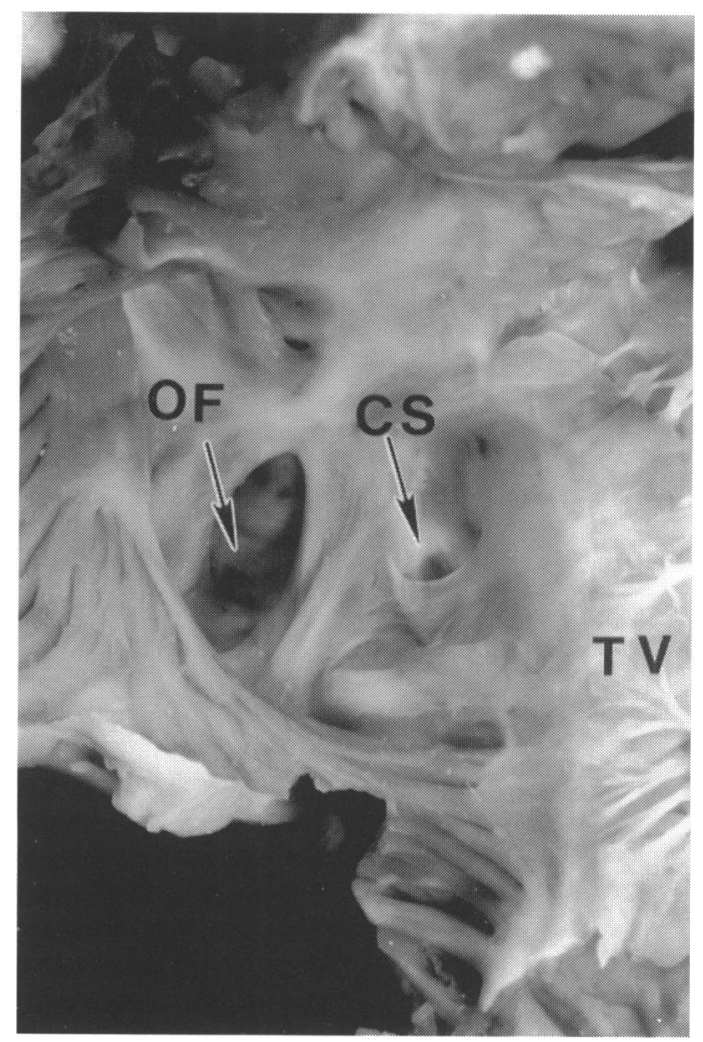

Figure 1 A normal heart viewed from the right atrium showing the floor and the rims of the oval fossa. CS coronary sinus; $O F$, oval fossa; $T V$, tricuspid valve. 
Figure 2 Dissections of adult human hearts showing the components and extent of the atrial septum. (A) Viewed from behind having removed the atrial domes and cleaned the fibrofatty tissue from

the interatrial groove.

(B) Frontal section. Both hearts show the deep infolding (interatrial groove) that produces the so-called "septum secundum", in reality an inflexion of the parietal walls of the atrial chambers.
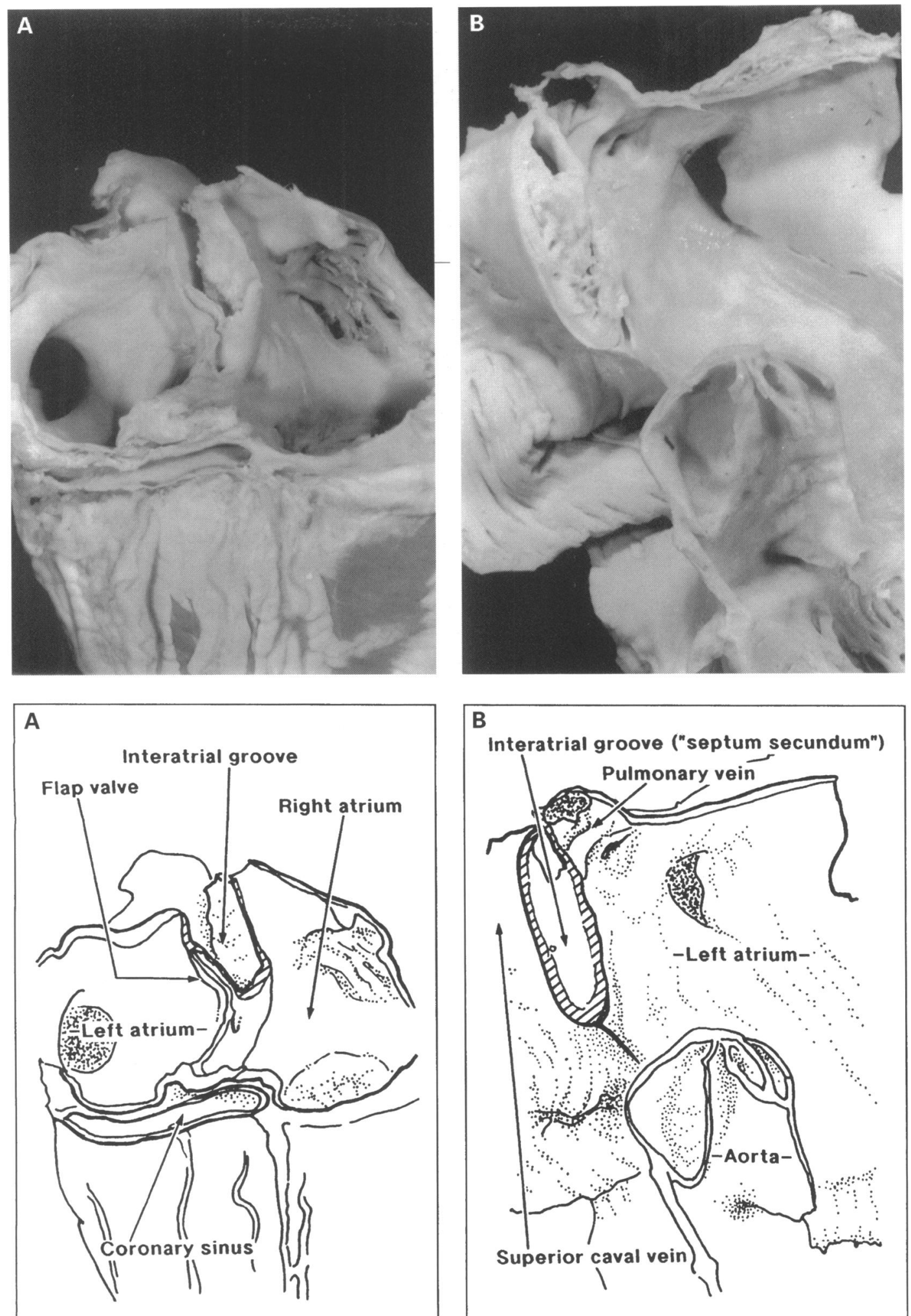

Furthermore, despite these various descriptions, it remains a fact that their location makes them difficult to identify and diagnose correctly during life. Despite prior description of the diagnostic echocardiographic features for both varieties, ${ }^{7-9}$ false positive and false negative findings continue. Indeed, a recent echocardiographic study ${ }^{10}$ failed even to discuss the extraseptal nature of the communications. To establish the potential reasons for diagnostic fallibility, we reviewed the echocardiograms, cine-angiocardiograms, and surgical notes of all the patients in whom a preopera-

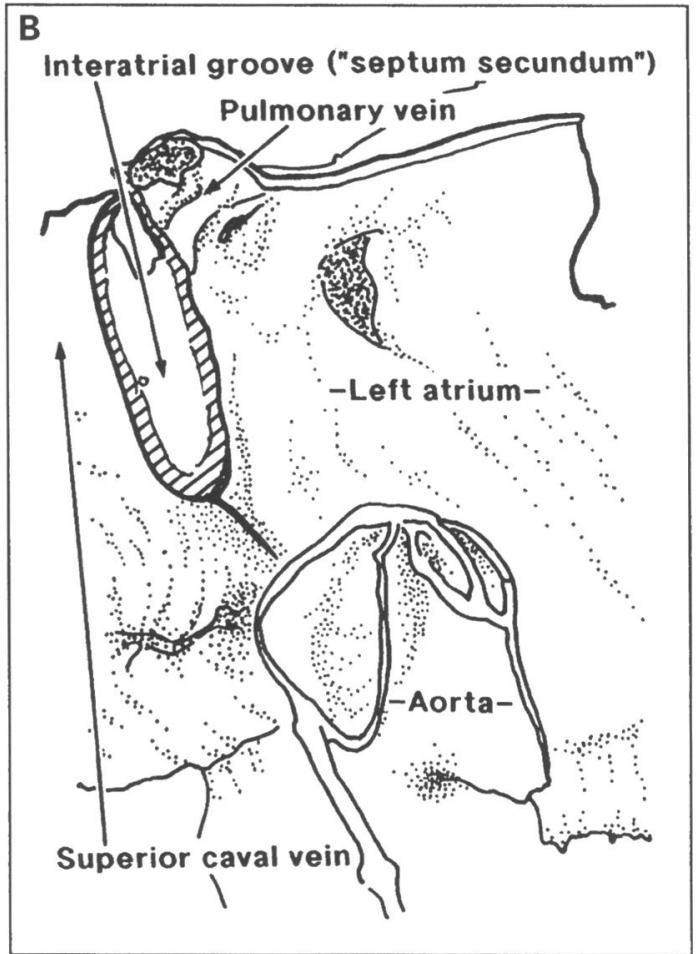

tive diagnosis of sinus venosus defect had been made at the Royal Brompton Hospital in London between July 1991 and August 1996. We judged the clinical findings on the basis of anatomical study of five autopsied specimens purported to have sinus venosus defects, together with examination of the normal atrial septal structure.

\section{Patients and methods}

Echocardiograms and surgical notes were reviewed from 18 patients in whom a preoper- 
Figure 3 Pathological specimen of superior sinus venosus defect viewed from the right atrium. The defect is outside the confines of the oval fossa, with the mouths of the overriding superior caval vein having a biatrial connection and overriding the rim of the fossa. The right pulmonary vein is connected to the superior cavoatrial junction. CS, coronary sinus; $O F$, oval fossa; $P V$, right upper pulmonary vein; SCV, superior caval vein; $T V$, tricuspid valve; SSVD, superior sinus venosus defect.

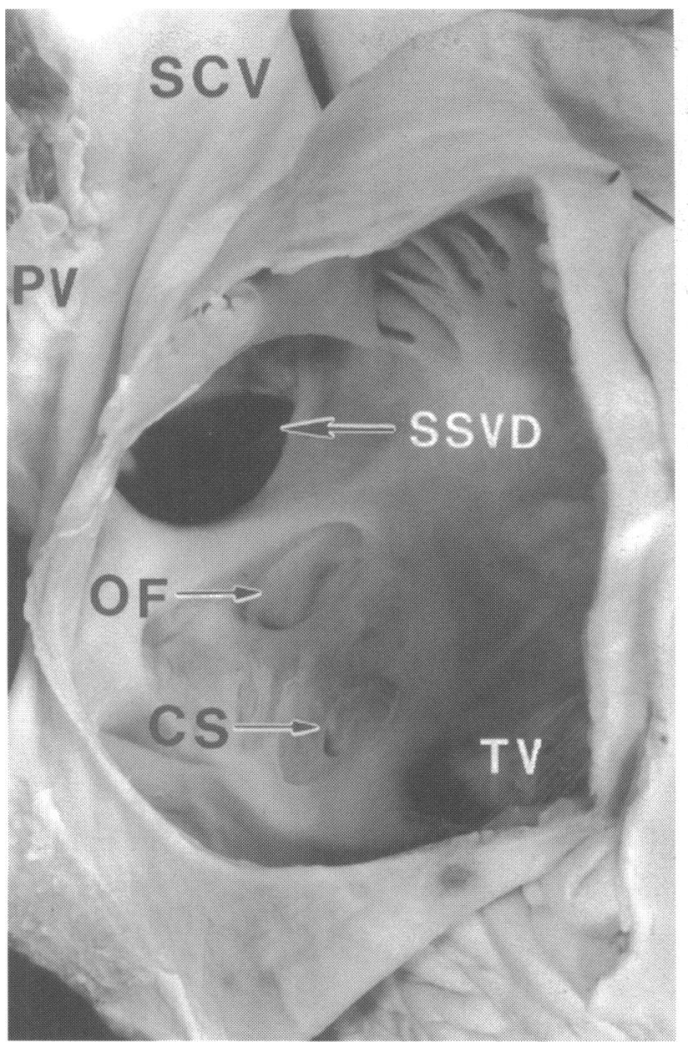

ative diagnosis of a sinus venosus defect had been made over the five year study period. Successful surgical correction has been achieved in 17 patients. In all patients, the junctions of the superior and inferior caval veins with the atrial chambers and the atrial septum, together with the pulmonary venous connections, had been evaluated carefully echocardiographically from the subcostal window. Colour flow mapping had also been performed using a Hewlett Parkard 1000 sector scanner. Four patients had undergone transoesophageal examination, and cardiac catheterisation had been performed in two patients.

Five specimens reputed to show sinus veno-

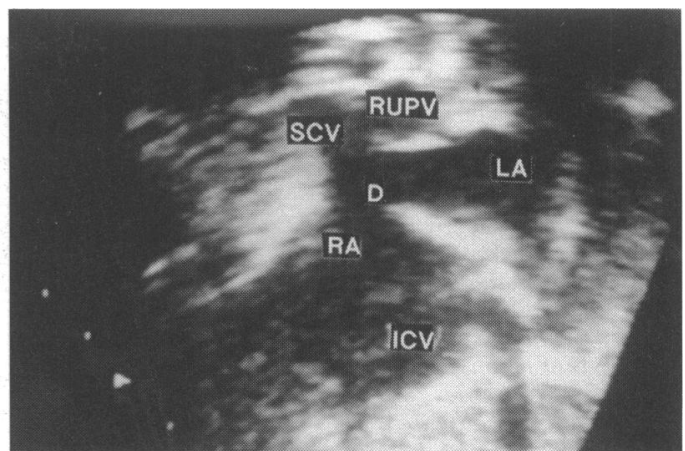

Figure 4 Cross sectional echocardiogram of case 11 with superior sinus venosus defect, taken in the subcostal long axis sagittal plane. A large interatrial communication is formed by the month of the overriding superior caval vein. The anomalous right upper pulmonary vein is connected to the superior cavoatrial junction. $D$, defect; $I C V$, inferior caval vein; $L A$, left atrium; $R A$, right atrium; $R U P V$, right upper pulmonary vein; $S C V$, superior caval vein.

sus defects were available for study in the cardiopathological museum of the National Heart and Lung Institute in London, three showing the superior variant of sinus venosus defect and two initially thought to be of the inferior variety. All examples of the superior variant had come from the collection of the National Heart Hospital established by $\operatorname{Dr}$ R E B Hudson. The findings in the abnormal hearts were supplemented by examination of the atrial septal structure in 10 normal adult hearts.

\section{Results}

MORPHOLOGICAL FINDINGS

In the normal heart, the tissues separating directly the cavities of the right and left atrial chambers are found in the floor and inferior border of the oval fossa (fig 1). The floor of the fossa is a relatively thin fibromuscular flap that, anterosuperiorly, overlaps an apparently extensive muscular border, being firmly fused to the border in the majority of hearts. The posterior border of the fossa continues directly

Echocardiographic and surgical findings in patients with preoperative diagnosis of sinus venosus defects

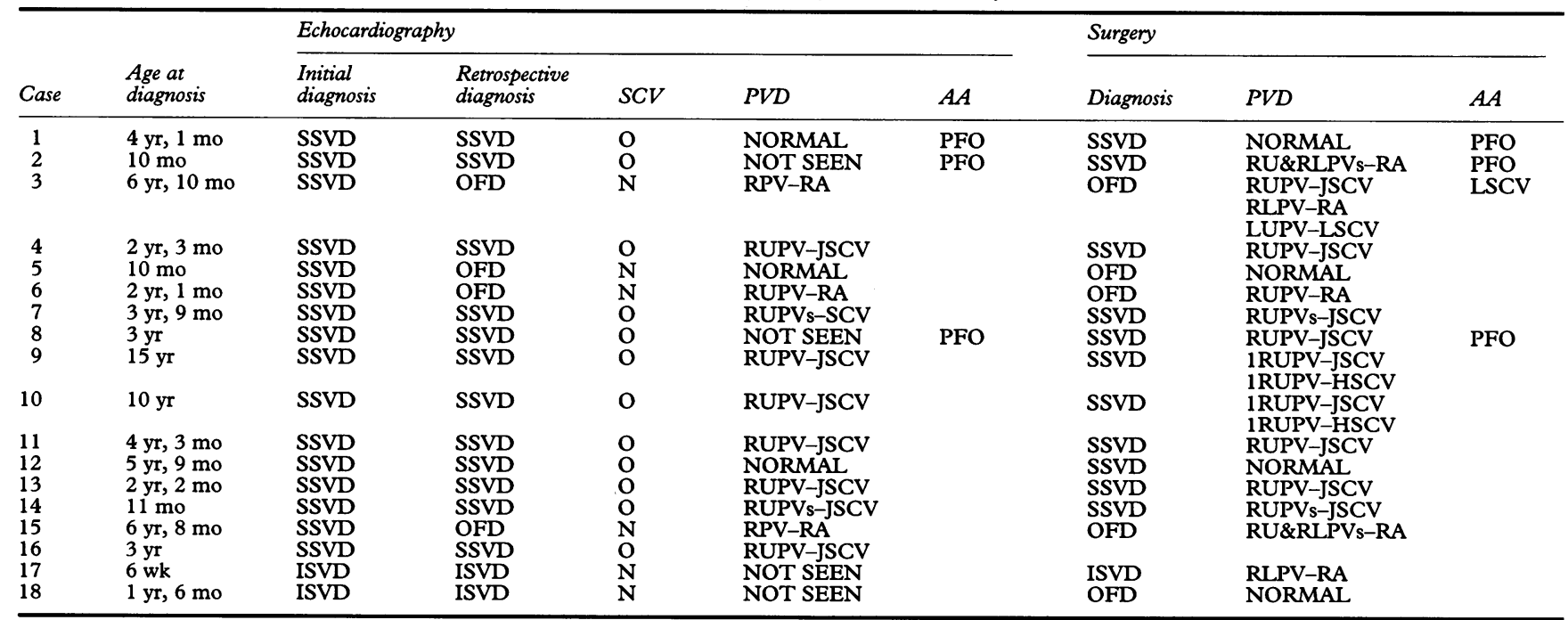

AA, associated anomalies; HSCV, high of superior caval vien; ISVD, inferior sinus venous defect; JSCV, junction of superior caval vien; LSCV, left superior caval vien; LUPV, left upper pulmonary vein; N, no overriding; $O$, overriding; OFD, oval fossa defect; PFO, patent foramen ovale; RA, right atrium; RLPV, right
lover pulmonary vien; RUPV, right upper pulmonary vien; RPVD, right pulmonary veinous drainage; SCV, superior caval vein; SSVD, superior sinus venosus defect. 
Figure 5

Transoesophageal echocardiogram of case 16 with superior sinus venosus defect showing overriding of the superior caval vein upper pulmonary vein connected to the superior cavoatrial junction. $L A$, left atrium; $R A$, right atrium; $R P V$, right pulmonary vein; SCV, superior caval vein.

Figure 6 Surgical findings of case 15 showing defects within the oval fossa and anomalous drainage of right upper and lower pulmonary veins to the sinus; $O D$, oval fossa defects; $R P V$, right tricuspid valve. and an anomalous right right atrium. $C S$, coronary pulmonary veins; $T V$
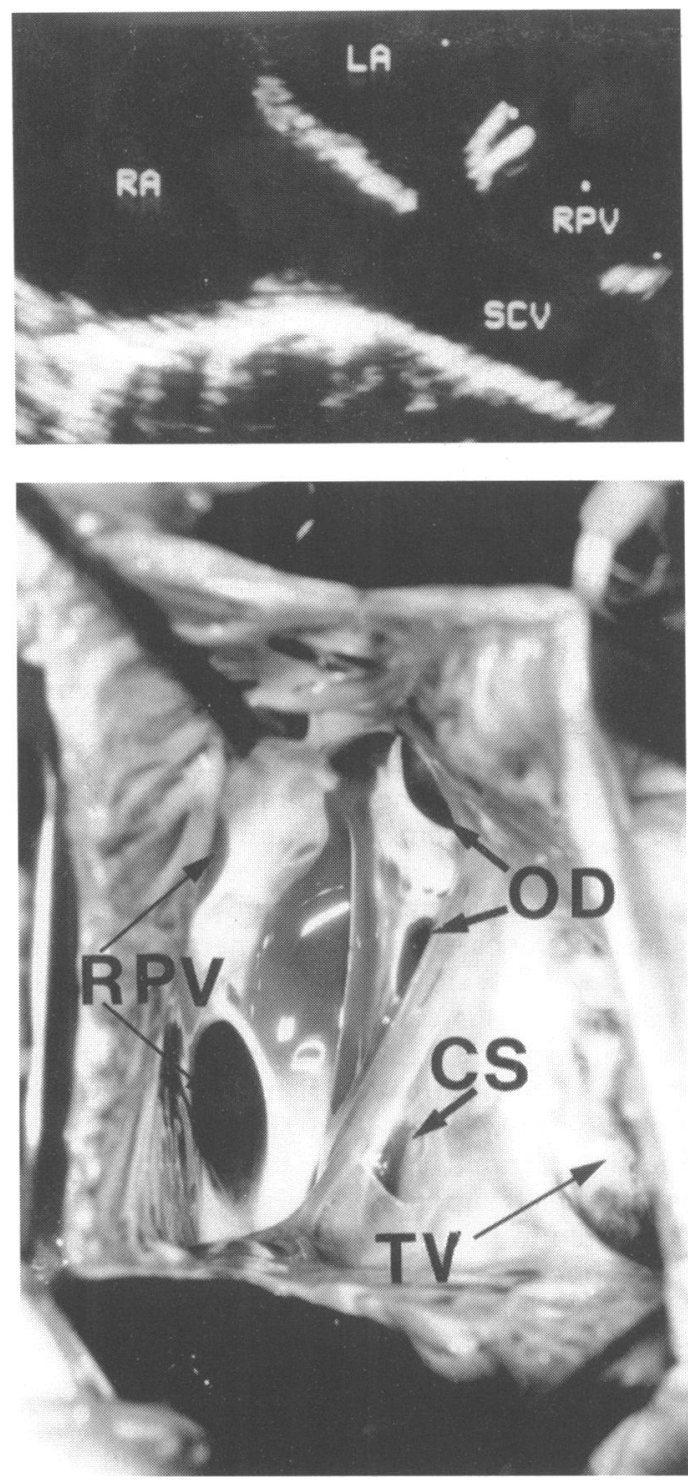

into the wall of the inferior caval vein. The inferior border of the fossa is continuous with the muscular atrioventricular septum, the latter representing the area between the offset hinge points of the tricuspid and mitral valves.
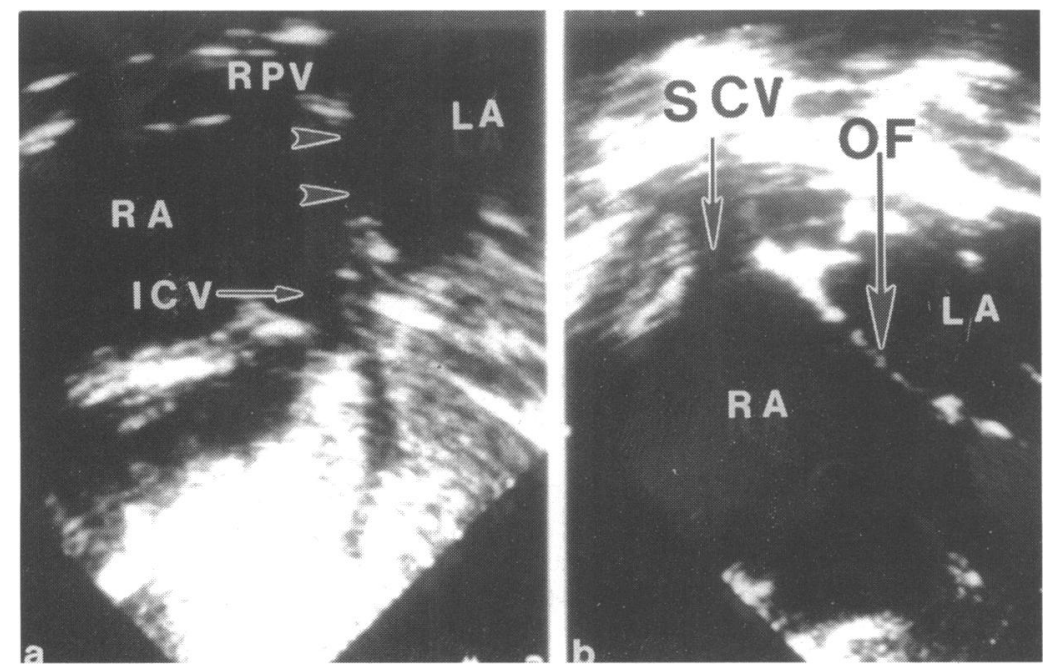

Figure 7 Cross sectional echocardiogram from the subcostal window of case 17 diagnosed as having an inferior sinus venosus defect. The large interatrial communication (a) is located inferiorly and posteriorly, adjacent to the atrial connection of the inferior caval vein (arrow head). Other cuts (b) show the intact oval fossa (OF). ICV, inferior caval vein; $L A$, left atrium; $R A$, right atrium; $R P V$, right pulmonary vein; $S C V$, superior caval vein.
The anterosuperior border of the fossa, in contrast, is formed by a deep inflexion of the walls of the two atria. This is particularly marked superiorly, where there is an extensive fold between the attachments of the superior caval vein to the right atrium and the right pulmonary veins to the left atrium (fig 2). It should be noted that, throughout the extent of this fold, which is filled by extracardiac adipose tissue, the thickness of the walls is comparable with that found in the parietal atrial components.

In all three specimens with a superior sinus venosus defect, the orifice of the superior caval vein overrode the upper border of the oval fossa, with the walls of the vein continuous with those of both the right and left atria. The overriding mouth of the vein, therefore, forms a large interatrial communication outside the confines of the oval fossa (fig 3). In two of the hearts, the right upper pulmonary vein connected to the superior cavoatrial junction. In the third specimen, the right upper pulmonary vein was connected high to the superior caval vein, whereas the lower one was connected at the cavoatrial junction.

In both specimens thought initially to have inferior sinus venosus defects, the inferior caval vein did have a biatrial connection. Careful examination of both hearts, however, showed that the caval vein overrode the oval fossa, and was not separated from it by a discrete muscular border. Both hearts, therefore, represented oval fossa rather than sinus venosus varieties of interatrial communication. Surgical repair had been attempted in both and the atrial partitioning was appropriate in one. In the other heart, in contrast, the eustachian valve had been mistaken for the lower border of the defect, incorrect placement of the patch then leaving the inferior caval vein connected to the left atrium.

\section{CLINICAL FINDINGS}

The echocardiographic and surgical data of the 18 patients (six female, 12 male, aged 1.5 months to 15 years, median four years) are summarised in table 1 . A preoperative echocardiographic diagnosis of a superior sinus venosus defect had been made in 16 patients. Retrospective review showed an interatrial communication outside the oval fossa, with overriding of the superior caval vein across its upper rim, in 12 of these patients. This had been demonstrated from the subcostal view using the long axis sagittal plane in three patients (fig 4), and using the conventional coronal plane in the others. In three patients (cases 1,2 , and 8), patency of the oval fossa had been revealed by colour flow mapping. A right upper pulmonary vein connecting to the junction of the superior caval vein with the atrium was found in eight patients (cases 4, 7, 9-11, 13, 14, and 16). Among these, two patients (cases 7 and 14) had one additional right pulmonary vein connected to this junction. In two patients (cases 1 and 12), the right pulmonary venous drainage was normal, while it could not be positively identified in the other two (cases 2 and 8 ). Transoesophageal studies 
had confirmed the diagnosis in three of these patients (cases 9, 10, and 16). In 11 of these patients, the echocardiographic findings were subsequently confirmed at surgical correction. The surgical inspection revealed further anomalous pulmonary venous connections in four patients, in one (case 8) to the junction, in one (case 2) directly to the right atrium, and in two (cases 9 and 10) to the superior caval vein itself. In case 9, a gusset was needed to enlarge the superior cavoatrial junction. Case $16 \mathrm{had}$ not undergone surgery at the time of writing. His transoesophageal echocardiogram demonstrated clearly biatrial connection of the superior caval vein, but with subtle overriding across the upper rim of the intact oval fossa. The right upper pulmonary vein was shown to be connected to the superior cavoatrial junction (fig 5).

In four patients (cases 3, 5, and 6 presenting in 1991, and case 15 in 1996), overriding of the superior caval vein was not found during retrospective study of the echocardiograms, even though the initial diagnosis had been of a sinus venosus defect. In all these patients, surgery revealed that the large interatrial communication was within the oval fossa. Markedly anomalous pulmonary venous connections were found in case 3 , with the right upper pulmonary vein connected to the superior cavoatrial junction, the right lower pulmonary vein directly to the right atrium, and the left upper pulmonary vein to a left superior caval vein that, in turn, connected to the right atrium. Transoesophageal echocardiography in case 15 revealed two defects within the oval fossa, and demonstrated connection of the anomalous right pulmonary veins to the right atrium, but excluded the overriding of the superior caval vein. Angiocardiography in this case was also interpreted as showing a large atrial septal defect within the oval fossa, with the right upper and lower pulmonary veins connecting directly to the right atrium. The surgical findings confirmed this diagnosis (fig 6).

Two patients (cases 17 and 18) had been diagnosed preoperatively as having inferior sinus venosus defects. Retrospective study of the echocardiograms revealed a large interatrial communication located inferiorly and posteriorly, adjacent to the atrial connection of the inferior caval vein (fig 7a). The right pulmonary veins could not be identified in either patient but the intact nature of the oval fossa could be demonstrated in one (fig $7 \mathrm{~b}$ ), albeit that the tracing obtained did not show the septum and the interatrial communication in the same cut. In only one of the patients (case 17) did the surgical findings confirm a biatrial connection of the inferior caval vein, with the right lower pulmonary vein connected to the inferior cavoatrial junction. No details were given, however, as to the nature of the rim of the oval fossa. In case 18 the defect was located posteriorly and inferiorly, adjacent to the mouth of the inferior caval vein, but with normal pulmonary venous connections.

Case 2 has developed right pulmonary venous obstruction at the site of the patch placed to re-route the veins from the right into the left atrium. This was noted five years after surgical correction. No arrhythmic or other complications were encountered at surgery or thus far in follow up in any of the other 16 patients who have undergone surgical correction, which was successful in all.

\section{Discussion}

The diagnosis and even the very nature of "sinus venosus interatrial communications" remain a matter of debate. ${ }^{1-9}$ Our experience shows that, even with modern day cross sectional echocardiography reinforced by colour flow mapping, preoperative diagnosis does not always concur with findings at surgery. Thus, in four of our patients predicted preoperatively to have sinus venosus defects, the surgeon found large defects within the oval fossa, albeit with markedly abnormal connection of the pulmonary veins to the morphologically right atrium in three cases. Similarly, it proved difficult preoperatively to find reliable echocardiographic evidence of the precise location of the presumed inferior sinus venosus defects. This experience, therefore, begs the question of the very nature of the sinus venosus defect and, indeed, the precise nature of the atrial septum. Distinction of the septal boundaries is crucial in providing solid criteria for diagnosis. In our opinion, a septal structure represents that part of the walls of the heart that can be removed without transgressing on the pericardial cavity. ${ }^{11}$ When defined in this way, we are able unequivocally to distinguish between septa and parietal structures. Parietal structures can be infolded to produce a partition between adjacent chambers, but such a partition has two layers, and incorporates an intervening layer of extracardiac space. Definition of the atrial septum following this principle, namely as that part of the atrial walls that can be removed without leaving the heart, shows that the area occupied by the septum is much smaller than proposed by previous investigators. ${ }^{12}$ Much of the extensive crest depicted by these authors ${ }^{12}$ as visible from inside of the right atrium represents the infolded atrial walls, as shown by the sections illustrated in figure 2. Recognition of the extent of the septum, and distinction of infolded borders as opposed to true septa, is important when making the distinction between atrial septal defects and interatrial communications. ${ }^{13}$ This may seem a play on words but, in reality, is of major diagnostic significance. Atrial septal defects, in other words deficiencies of the true atrial septum, are produced by deficiency of all or part of the flap valve of the septum. Such defects are, of necessity, within the confines of the muscular borders of the oval fossa. Interatrial communications self evidently include such true defects of the atrial septum. Other anatomical interatrial communications, in contrast, can permit shunting of blood at atrial level, but outside the confines of the oval fossa. Such communications outside the confines of the true septum can take the form of coronary sinus defects, atrioventricular septal 
defects of "ostium primum" variety, and, of particular interest in the present context, sinus venosus defects.

These morphological studies confirm previously expressed opinions, ${ }^{78}$ namely that the anatomical prerequisite for existence of a sinus venosus defect is overriding of the intact muscular border of the oval fossa by the mouth of the superior or inferior caval vein. It is the overriding and consequent biatrial connection of the superior versus the inferior caval vein that distinguishes the two types of sinus venosus defects. Such biatrial connection can be remarkably subtle, as demonstrated by case 16 , but is always in our experience to be seen following careful examination. In most, but not all, of our cases the connections of the right pulmonary veins were also abnormal, but abnormal pulmonary venous connections are not a prerequisite for diagnosis. It is the presence of the defect outside the confines of the oval fossa that is the key to diagnosis. Thus, we suggest that a positive diagnosis of sinus venosus defect can be made only when the oval fossa itself is shown to have intact rims. A deficiency within the oval fossa can exist with a sinus venosus defect, but it is recognition of the intact muscular border surrounding the fossa, being overridden to various extent by the caval vein, that permits unequivocal diagnosis.

This criterion is valuable not only for the morphologist and echocardiographer but also for the surgeon. In the case of the inferior sinus venosus defect, use of this criterion begs the question as to the distinction between a true extraseptal communication which has become confluent with the oval fossa, as opposed to an extensive deficiency within the fossa extending to reach the mouth of the fossa. There is no answer to this potential discrepancy, reinforcing our opinion that the sinus venosus defects should be identified only when the oval fossa itself can be shown to have intact borders. This can be difficult to establish echocardiographically; however, on this basis, it proved necessary to re-classify both our morphological specimens as having defects within the oval fossa. We suggest that this approach should also be followed in clinical evaluation. Of much more practical importance in this regard is the need for the surgeon to distinguish between the right and left atrial margins of the overriding mouth of the inferior caval vein. Unless suitable care is taken, the surgeon may find that, as in one of our cases, the surgical repair results in the inferior caval vein being connected to the left rather than the right atrium. This happens when the patch is sewn to the eustachian ridge in the right atrium, as opposed to the wall of the inferior caval vein which is continuous with the left atrial myocardium when its mouth is overriding.

Such surgical manoeuvers are less problematic with the superior variety of defects, although it can still prove difficult properly to separate the venous streams without producing obstruction. In the past, it was often considered necessary to enlarge the superior caval venoatrial junction by inserting a prosthetic gusset. ${ }^{14}$ Such a procedure places at risk either the sinus node or its arterial supply. It was necessary in only one of our patients to enlarge surgically the venoatrial junctions. Rigorous avoidance of the area known to harbour both the sinus node and its arterial supply at cannulation and when placing sutures to secure the patches used for separation, has meant that, thus far, our patients have been free from arrhythmic complications. One patient did develop postoperative pulmonary venous obstruction over a period of five years.

Although, in our opinion, it is the presence of an abnormally located caval vein attached to both atria that is the key to diagnosis, others have used alternative criteria ${ }^{56}$ for the existence of a sinus venosus defect. The alternative approach is based largely upon developmental premises that, thus far are unsubstantiated by anatomical facts. In particular, our study offers no evidence to substantiate the concept of malposition of the primary atrial septum..$^{56}$ The way in which sinus venosus defects are produced during fetal life is unknown. Until we have direct embryological evidence bearing on these events, it seems much more sensible to use anatomical criteria for diagnosis. This is heightened as debate continues among embryologists concerning the extent and boundaries of the embryonic venous sinus (sinus venosus). In an excellent study performed several decades ago, Auer produced convincing evidence that the portal of entry of the developing pulmonary vein to the heart was morphologically distinct from the tributaries of the systemic venous sinus. ${ }^{1516}$ More recently, these findings have been questioned by other investigators who argue that the pulmonary vein is connected to a component of the embryonic systemic venous sinus that then becomes incorporated in the developing left atrium. ${ }^{17} 18$ In our opinion, the criteria for definition of the systemic venous sinus used by the latter investigators are not nearly as convincing as those used by Auer. ${ }^{1516}$ Furthermore, while the hypothesis of incorporation of part of the systemic venous sinus into the morphologically left atrium is initially appealing to explain the sinus venosus defect, it is difficult to understand how this can also be reconciled with normal formation of the borders of the oval fossa, a prerequisite for diagnosis. This reinforces our opinion that we should rely currently on an anatomical criterion for diagnosis, namely, overriding of the intact muscular border of the oval fossa by the abnormally located mouth of one or other of the caval veins.

We are indebted to Professor Andrew Redington and Dr Elliot Shinebourne for permission to include patients under their care; to Mr Manjit Josen who performed many of the echocardiogernicierable help in preparation of the illustrations. RHA is supported by the British Heart Foundation together with the Joseph Levy Foundition.

1 Abbott ME. Congenital cardiac disease. In: Osler W, McCrae T, eds. Modern medicine-its theory and practice. Philadelphia: Lea and Febiger, 1907:349.

2 Ross DN. The sinus venosus type of atrial septal defect. Guy's Hosp Rep 1956;105:376-81.

3 Swan HJC, Kirklin JW, Becu LM, Wood EH. Anomalous connection of right pulmonary veins to superior vena 
cava with interatrial communications. Hemodynamic data in eight cases. Circulation 1957;16:54-66.

4 Lewis FJ, Taufic M, Vacro RL, Niazi S. The surgical anatomy of atrial septal defects: experiences with repair under direct vision. Ann Surg 1955;142:401-17.

5 Van Praagh S, Carrera ME, Sanders SP, Mayer JE, Van Praagh R. Sinus venosus defects: unroofing of the righ pulmonary veins - anatomic and echocardiographic findings and surgical treatment. Am Heart $\mathcal{F}$ 1994;128:

6 Van Praagh S, Carrera ME, Sanders SP, Mayer JE, Van Praagh R. Sinus venosus defect [letter]. Am Heart $\mathcal{F}$

7 Ettedgui JA, Siewers RD, Anderson RH, Park SC, Pahl E Zuberbuhler JR. Diagnostic echocardiographic features of the sinus venosus defect. $B r$ Heart $\mathcal{F}$ 1990;64:329-31.

8 Ettedgui JA, Siewers RD, Zuberbuhler JR, Anderson RH. Echocardiographic diagnosis of inferior sinus venosus defect. Cardiol Young 1992;2:328-41.

9 Muhler EG, Engelhardt W, Von Bernuth G. Detection of sinus venosus atrial septal defect by two-dimensional echocardiography. Eur Heart $₹$ 1992;13:453-6.

10 Pascoe RD, Oh JK, Warnes CA, Danielson GK, Tajik AJ, Seward JB. Diagnosis of sinus venosus atrial septal defect seward JB. Diagnosis of sinus venosus atrial septal defect
with transesophageal echocardiography. Circulation 1996;
94:1049-55

11 Anderson RH, Brown NA. The anatomy of the heart revisited. Anatomica Record 1996;246:1-7.

12 Sweeney LJ, Rosenquist GC. The normal anatomy of the atrial septum in the human heart. Am Heart $₹ 1979 ; 98$ : 194-9.

13 Wilcox BR, Anderson RH. Surgical anatomy of the heart. 2nd edn. London: Gower Medical, 1992:7 6-7.7.

14 Kyger ER, Frazier OH, Coodey DA, Gillette PC, Reul GJ, Sandiford FM, et al. Sinus venosus atrial septal defect: early and late results following closure in 109 patients. Ann Thorac Surg 1978;25:44-50.

15 Auer $\mathrm{J}$. The early development of the sinu-atrial region in the rat. Acta Neerland Morph 1941;4:214-32.

16 Auer J. The development of the human pulmonary vein and its major variations. Anat $\operatorname{Rec} 1948 ; 101: 581-94$

17 DeRuiter MC, Gittenberger-de Groot AC, Wenink AC, Poelmann RE, Mentink MM. In normal development pulmonary veins are connected to the sinus venosus segment in the left atrium. Anat Rec 1995;243:84-92.

18 Tasaka H, Krug EL, Markwald RR. Origin of the pulmonary venous orifice in the mouse and its relation to the morphogenesis of the sinus venosus, extracardiac mesenchyme (spina vestibuli), and atrium. Anat Rec 1996; 246:107-13. 